\title{
End-growth results in juvenile idiopathic scoliosis treated with conservative approach
}

\author{
Claudia Fusco ${ }^{*}$, Fabio Zaina, Stefano Negrini \\ From 7th International Conference on Conservative Management of Spinal Deformities \\ Montreal, Canada. 20-22 May 2010
}

\begin{abstract}
Background
According to SRS (Scoliosis research Society) Juvenile idiopathic scoliosis (JIS) is defined as a scoliosis that is first diagnosed between the age of 4 and 10 years. The natural history is characterized by an early deformity that leads to a major but extremely variable progression risk throughout growth. Objectives of the study is verifying the efficacy of a complete conservative treatment of juvenile idiopathic scoliosis.
\end{abstract}

\section{Materials and methods}

Observational retrospective study. Population: we included all JIS patients for which we have consecutive radiographies from Risser test 0 and to 3 since our database start in 2003. We had 30 patients, 27 females and 3 males; mean age at start $7.8 \pm 1.5$, mean treatment lasting 5,8 years. For 23 of 30 patient studied we have also radiography at Risser 5 and we evaluated radiographic evolution from Risser 3 to Risser 5. Finally we split patient in 3 groups according to curve degrees at first diagnosis: 9 patients presented a curve under $20 \mathrm{Cobb}$, 11 patients between 20 and $30^{\circ}$ Cobb and 7 over $30^{\circ}$ Cobb. Methods: according to individual needs patient were treated with physical exercises alone or with brace and physical exercises. Outcome criteria: radiographic (Cobb degrees) and clinical (ATR, hump, TRACE,).

\section{Results}

Mean thoracic Cobb degrees values changed with treatment from $24.4 \pm 10.0$ to $25.8 \pm 11.2$, thoracolumbar from $1.7 \pm 7.4$ to $1.0 \pm 4.1$ and lumbar from $17.9 \pm 10.4$ to $18 \pm 11.7$. We have not statistically significant differences in the Cobb degrees and also in the evaluation of the hump. $6.6 \%$ of patients started with a curve over $45 \mathrm{Cobb}$ degrees and improved during the treatment.
While $33.3 \%$ worsened at end of treatment more than $5 \mathrm{Cobb}$ degrees. In the subset of patients under $20^{\circ} \mathrm{Cobb}$ and between 20 to $30^{\circ} \mathrm{Cobb}$ we observed a consistent but not significant change while in the group of patient that started treatment over $30^{\circ}$ we observed a non significant improvement. In 23 cases that reached end of growth (for which we have radiography at Risser 5) we observed a maintenance of results reached at Risser 3.7 of $23(30.4 \%)$ patient arrived at end of treatment with a curve over $35^{\circ}$ and 2 of 23 .

\section{Discussion}

JIS may have a variable behaviour but generally it shows a progressive worsening during the year. Treatment can face this natural worsening trend. We observed a good corrective power of brace in the first years of treatment until Risser test 1 with a decrease of correction in the following years and a maintenance of achieved correction.

\section{Conclusion}

Conservative treatment start in childhood may favourable changes natural history of scoliosis. Prospective studies are needed to better investigate this particular kind of scoliosis.

Published: 10 September 2010

doi:10.1186/1748-7161-5-S1-O71

Cite this article as: Fusco et al:: End-growth results in juvenile idiopathic scoliosis treated with conservative approach. Scoliosis 2010 5(Suppl 1): 071. 